\title{
Entre la invasión y la consolidación de barrios: análisis psicosocial de la resistencia al desalojo ${ }^{1}$
}

\author{
Esther Wiesenfeld \\ Universidad Central de Venezuela
}

\begin{abstract}
Resumen
En el presente trabajo ilustraremos algunos procesos mediante los cuales los habitantes del barrio se ven sometidos simultáneamente a prácticas de inclusión y exclusión por parte de las políticas estatuidas, lo cual genera contradicciones en el seno de las instituciones oficiales así como en los pobladores expuestos a las mismas. Analizaremos, a partir de un estudio de caso, estrategias empleadas por los pobladores para enfrentar las amenazas de desalojo a las que recurrentemente se ven expuestos y sugeriremos que las soluciones al problema habitacional autogestionado por los propios ciudadanos, plantean entre otros, el dilema entre la necesidad y la legalidad.

Descriptores: Psicología Ambiental, Barrio, Resistencia al desalojo.
\end{abstract}

Key-words: Environmental Psychology, "Barrio",

Displacement resistance.

\section{Abstract}

Between the invasion and consolidation of "Barrios": psicosocial analysis of displacement resistance. In the present paper we will illustrate some of the processes by which the habitants of a "Barrio" are simultaneously subject to practices of inclusion and exclusion coming from the state policies. This generates contradictions in the backbone of the official institutions same as in the habitants exposed to such policies. We will analyze, stemming from a case study, strategies employed by the habitants for facing the threats of displacement to which they see themselves constantly exposed and we will suggest that the solutions to housing problems conducted and solved by the citizens themselves, pose among other things, the dilemma between necessity and legality. 
“...algo esencial que se conoce también como «comunidad», se eclipsa con el acceso a las formas urbanas de la opulencia tal como ahora están las cosas."

(Sennett, 1975:19)

\section{I. ¿Cómo surgen los asentamientos urbanos no regulados?}

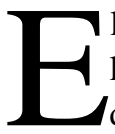

1 surgimiento de los asentamientos urbanos no regulados, llamados barrios en Venezuela, está asociado a las condiciones de pobreza de sus habitantes, quienes al no poseer los recursos necesarios para optar al alquiler o compra de viviendas construidas de acuerdo a la normativa vigente y en sectores urbanizados de las ciudades, optan como alternativa última asentarse en terrenos municipales o privados, y construir allí su albergue.

El mito de la opulencia en las ciudades unido a las precarias condiciones de vida en el campo, atrae a estos ciudadanos a las zonas urbanas, tendencia ésta cuyo incremento en el último cuarto de siglo ha invertido la distribución de la población rural-urbana latinoamericana (Ruibal, 1993), previéndose que para el año 2.000 el 80\% de los latinoamericanos vivirán en ciudades (Jiménez, 1993).

Por su parte Lovera (1989) señala que el 30\% de la población total y el 50\% de la urbana vive en barrios de ranchos, cifras que tienden a incrementarse con la crisis del país, estimándose que en el año 2000 el 85\% de la población del área metropolitana de Caracas residirá en ese tipo de asentamientos y que las condiciones de vida en los mismos empeorarán. Esta concentración poblacional y de la pobreza, actual y potencial, en las ciudades latinoamericanas, y específicamente en nuestro país, ha generado lo que Schteingart (1993) ha llamado "el mundo de la informalidad", el cual se caracteriza por "la presencia de escasas remuneraciones, empleos ocasionales y grandes cinturones de miseria donde prolifera la vivienda precaria, sin servicios y con niveles de vida deficientes" (p. 57)

Según Negrón (1993) existe una paradoja entre lo que se ha llamado "ciudad formal" y "ciudad informal". Este arquitecto sustenta su planteamiento en el hecho de que aunque la mayoría de la población 
habita en la ciudad informal, es excluida del orden urbano, es decir, de las normas que regulan la apropiación y uso del espacio urbano, lo cual supone para el autor una atribución implícita hacia el sector informal como anómalo, y por ende proclive a ser erradicado.

Para otros analistas del tema, la anomalía radica en la ilegalidad en la que incurren tanto los que se apropian ilegalmente del suelo como los organismos oficiales que con frecuencia simulan ignorar estas ocupaciones (Duhau, 1993).

En efecto, existe una brecha entre las prescripciones del derecho urbano y las prácticas de amplios sectores de la población, que da lugar a la constitución de una legalidad diferente, a través de los derechos mutuamente reconocidos por los sectores involucrados en los procesos de urbanización irregular (Panizzi, 1988, citado por Duhau, 1993). Los ciudadanos, al ver limitada su posibilidad de incorporar su voz en el discurso del poder estatuido, ejecutan prácticas reveladoras de formas alternativas de poder, lo que implica un derecho creado al margen del Estado. Por otra parte, el Estado legaliza tácitamente algunas de estas prácticas, llegando en ocasiones a implementar programas de asistencia técnica y a proveer recursos para mejorar la apariencia de las viviendas, el barrio, los servicios, pero en otras a desalojar por la fuerza a los pobladores y a destruir sus viviendas, cuando los terrenos van a ser destinados a un uso más lucrativo, o cuando su inestabilidad geológica amenaza la vida de sus ocupantes.

Las ambigüedades a las que están expuestos los agentes involucrados los impacta de manera diferente, lo cual tiene que ver con su reconocimiento de que la situación planteada representa a la vez un problema y una solución.

Las autoridades gubernamentales con frecuencia se debaten entre su deber de velar por el cumplimiento del orden y el respeto de la propiedad privada y su empatía con la necesidad de albergue del prójimo al cual el Estado no puede dar respuesta; entre preservar su estabilidad laboral o arriesgarla por su solidaridad con familias que tienen una trayectoria y una experiencia vivida en su barrio, que se han apropiado física y psicológicamente de ese lugar y que han hecho 
de él su hogar; en fin, se debaten entre su conciencia del deber y su conciencia del derecho a la vivienda que tienen los sectores afectados.

Para el residente, los esfuerzos físicos, materiales y psicológicos invertidos en la lucha por la estabilidad residencial, coexisten con la zozobra ante el riesgo de derrumbe o de desalojo de su vivienda, lo cual lo obliga a vivir en un estado de incertidumbre, provisionalidad, de anticipación de movilidad, cuando lo que anhela es estabilidad.

De acuerdo con Guitián (1993), el proyecto "barrio" tiene posibilidades en la medida en que se reconozcan tanto las limitaciones como las potencialidades naturales y sociales exógenas y endógenas. Las potencialidades naturales se refieren a las condiciones del terreno, las sociales exógenas, a la actitud conciliatoria del Estado y las instituciones para avalar implícitamente estos desarrollos habitacionales y las sociales endógenas a los mecanismos para enfrentar, ya sea a título individual o grupal, las situaciones de riesgo que acompañan soluciones como las planteadas. Las limitaciones naturales se reflejan en las irregularidades y riesgos que involucra la construcción en terrenos ubicados en zonas geológicamente inestables; las sociales exógenas estarían dadas por los riesgos posibles de la invasión (el desalojo) y las endógenas por las carencias de la población autoconstructora.

La información que predomina en torno a la situación descrita remite preferentemente a los problemas físico-estructurales, sociales y económicos en los asentamientos precarios y a datos sociodemográficos de sus habitantes, y proviene fundamentalmente de los organismos oficiales. Sin embargo, llama la atención el desconocimiento existente acerca de los modos de vida en los barrios y su impacto psicosocial sobre los pobladores, las formas que éstos tienen de interpretar ambos; es decir, existe un vacío de información relativo a la dimensión humana y social del tema, incluyendo los aspectos positivos de las comunidades que allí surgen. Este hecho, llamativo a la luz de la elevada proporción de la población que en ellos habita, nos motivó a iniciar una investigación orientada, entre otros objetivos, a conocer desde los protagonistas, las formas en las que éstos viven, sienten e interpretan su realidad.

Desde el punto de vista teórico, la investigación estuvo fundamentada en el paradigma construccionista (Gergen, 1985; Guba, 1990; 
Harré, 1989; Ibañez, 1989, 1994; Lincoln, 1994) el cual postula que la realidad es una construcción social porque se construye en la interacción a través del lenguaje y los significados compartidos; es múltiple porque los contextos de las interacciones cambian, y al hacerlo cambian los significados que se le asignan; está condicionada históricamente e influida por las condiciones socio-estructurales prevalentes en un determinado momento y lugar. Rescata la importancia de la dimensión subjetiva en dicha construcción, la cual también involucra al investigador, y destaca su naturaleza simbólica. En consecuencia, los objetivos de investigaciones guiadas por esta perspectiva teórica, tenderán a identificar los procesos a través de los cuales las personas construyen descripciones, explicaciones, valoraciones, de la realidad en la que viven; organizan su experiencia, conocimiento y transacciones relativas al mundo social y los interpretan a través de la identificación de los estados intencionales que las impulsan y por medio de los sistemas simbólicos de la cultura en la cual emergen, a saber, modalidades de lenguaje y discurso, formas de explicación lógica y narrativa, patrones de vida (Blumer, 1969; Bruner, 1991).

Dado que la interpretación es un proceso abierto, la construcción social de los objetos variará con nuestra exposición a nuevas situaciones y experiencias, de allí que sea concebido en tanto proceso antes que producto.

Estas características, trasladadas a nuestro foco de interés, se tradujeron en el análisis de los discursos que hombres y mujeres residentes en barrios construyen respecto a las formas de apropiación y usos de los espacios públicos y privados de aquél, en las distintas etapas de su desarrollo.

A continuación describiremos las características metodológicas que orientaron la investigación, parte de cuyos datos constituyen la base de este artículo.

\section{Contexto y características del estudio}

1.El contexto venezolano. La presencia de los barrios en el contexto social venezolano, tiene una larga data. En efecto, a partir de la década del cuarenta comenzó en el país un fuerte movimiento migratorio hacia las grandes ciudades, en particular, hacia su ciudad 
capital, Caracas. Es así como en una forma acelerada, la composición poblacional de Venezuela pasa a ser mayoritariamente urbana.

Esta situación generó una multiplicidad de problemas, en particular, los referidos a los servicios públicos, la escasez de vivienda, la atención médica, entre otros. Estos problemas eran, en muchos casos, resueltos por las propias personas, quienes se organizaron y trabajaron en pro de su solución. Así, los barrios emergen como una respuesta a los problemas de vivienda que se hacían cada vez más difíciles para quienes llegaban a las ciudades en busca de un lugar donde vivir y trabajar, en fin, donde mejorar sus condiciones de vida.

La ocupación inicial de un terreno baldío y la posterior autoconstrucción de las viviendas, ha sido la modalidad que ha signado el origen y posterior consolidación de los barrios que rodean las grandes ciudades venezolanas. La lucha constante por la permanencia en el terreno invadido, sujeto a derrumbes y a amenazas de desalojo, al tiempo que se trabaja permanentemente en la construcción y mejoramiento de la vivienda y los espacios comunes, es un proceso común en los barrios venezolanos. En la actualidad, a casi cincuenta años del inicio de este proceso, asistimos a la presencia cada vez mayor de los barrios en el paisaje de nuestras ciudades. En efecto, el nivel de densificación y consolidación de los barrios ha alcanzado niveles considerables. De la precariedad inicial en las construcciones quedan acaso pocas reminisencias. Actualmente, la casi totalidad de las viviendas son de bloque, con piso de cemento y la inmensa mayoría cuenta con servicios de aguas blancas y negras.

De igual forma podríamos decir que la construcción y consolidación de nuestros barrios no se remite solamente a sus aspectos físicos y estructurales. Tal como lo sustentan Bolívar y colaboradores (Bolívar, Guerrero, Rosas, Ontiveros, De Freitas, Arnal \& Sancio, 1994), cada barrio representa una determinada realidad social caracterizada por múltiples aspectos culturales e históricos, que devienen en una identidad propia y claramente diferenciable de los demás grupos que componen el tejido social venezolano. Veamos uno de estos casos.

2.El Barrio León Droz Blanco. La comunidad en la cual realizamos el estudio, es un claro ejemplo de los aspectos que hemos 
mencionado con anterioridad. Ubicado en una zona de clase media del área metropolitana de Caracas, el barrio tuvo su origen en la década del cincuenta, a partir de una invasión del terreno por parte de algunas personas que para el momento no tenían vivienda. Desde ese mismo momento, comienza la autoconstrucción colectiva de las viviendas, las cuales se realizaban en forma muy rápida y con materiales tales como cartón y latas, lo cual redundaba en una acentuada precariedad en este tipo de construcciones.

A medida que fueron llegando nuevos pobladores y se construyeron estas primeras viviendas, éstos tuvieron que organizarse para desarrollar todo tipo de estrategias que les permitiera adecuar el terreno a sus necesidades. Por otra parte, a lo largo de su historia, el barrio soportó no menos de cinco intentos de desalojo, tanto por parte de instituciones estatales así como por particulares interesados en utilizar los terrenos con fines de construcción privada. Sin embargo, estos intentos fracasaron una y otra vez, debido fundamentalmente al espíritu de organización y lucha que los residentes fueron cristalizando en el tiempo, con base en los vínculos sociales y afectivos construidos paralelamente a la construcción de sus vivienda y los servicios. En efecto, el nivel de consolidación alcanzado por la comunidad, tanto en lo que se refiere a sus aspectos estructurales como sociales, ha sido un elemento decisivo en cada instancia de amenaza que se ha vivido.

Actualmente, el barrio cuenta con 82 viviendas, en las que residen aproximadamente 400 personas. El mismo cuenta con todos sus servicios, sus viviendas han sido transformadas al límite de los tres pisos cada una y están dotadas de todos los servicios y comodidades posibles. Por otra parte, la población infantil y adolescente del barrio cuenta con un alto nivel de escolaridad y no existen ni han existido problemas epidemiológicos, todo lo cual indica el alto nivel de consolidación alcanzado por esta comunidad, originada en una invasión de un terreno baldío de la ciudad de Caracas.

3. Procedimiento. La información en la que basamos el análisis que presentamos a continuación proviene de una investigación que estuvo orientada a explorar la concepción de comunidad y a conocer 
el sentido de comunidad de los residentes de un barrio caraqueño (García \& Guliani, 1992), a analizar la importancia de la historia en la conformación y desarrollo de la comunidad (Giuliani, en prensa), y a conocer los procesos de construcción del significado de la casa y del barrio por parte de sus miembros (Wiesenfeld, en prensa).

Se decidió emplear la metodología cualitativa para la recopilación y análisis de la información, esto es, se abordó la investigación desde una perspectiva holística, sin hipótesis, variables ni teorías previamente definidas (Taylor \& Bogdan, 1986), sino pretendiendo dilucidar a través del análisis inductivo los procesos y conceptos mediante los cuales las personas entrevistadas construían el significado de los temas seleccionados, a saber: el proceso de construir sus casas, su barrio, su comunidad, y la interpretación que le asignaban a los mismos y a los fenómenos de su cotidianidad.

La información recopilada, derivó de diversas fuentes: a) la realización de entrevistas en profundidad con 14 residentes, ocho de sexo femenino y seis de sexo masculino y con edades comprendidas entre los 15 y los 76 años, b) la reconstrucción histórica de la comunidad a través de material archivado y de conversaciones informales individuales y grupales con los residentes del barrio, c) la realización de un taller con algunos miembros de la comunidad en el que se devolvió la información obtenida en las entrevistas y en la reconstrucción histórica del barrio a los asistentes y se propició una discusión crítica de los temas que emergieron en ambas.

\section{Análisis}

En primera instancia, es necesario aclarar que la exploración de los significados que tienen para las personas los tópicos investigados, se fundamentan en el punto de vista del propio actor, por lo que no hemos intentado determinar ni constatar una regularidad en la interpretación de las experiencias. No obstante, se hace evidente a través del análisis, un claro consenso acerca de las diferentes etapas a las que se vinculan algunos procesos, los cuales son posible identificar en los diferentes testimonios aportados por los entrevistados. Este ultimo punto se identificó a través de la elaboración de la historia de la comunidad, la cual fue discutida en conjunto con los miembros del 
barrio, quienes expresaron coincidencia acerca de la información generada a partir del trabajo.

Los resultados basados en las entrevistas realizadas permitieron identificar temas vinculados con las etapas del desarrollo de las viviendas y del barrio en el marco de los procesos que tienen lugar durante su consolidación, los cuales coexisten con la resistencia al desalojo. Los temas identificados fueron los siguientes:

1. Las diferentes fases del proceso constructivo de la vivienda. Este proceso se origina desde el momento inicial de la invasión al terreno y se continua en etapas sucesivas hasta su consolidación final. A lo largo del mismo fue posible observar, cómo a medida que la vivienda se transforma desde el punto de vista físico, también se transforman los significados que las personas les atribuyen. En ese sentido, se pueden establecer tres etapas en la evolución de estos aspectos. En la primera de ellas, se puede caracterizar a la vivienda como una construcción inicial sumamente precaria, construida en forma rápida, con materiales endebles o de desecho. A esa vivienda, los pobladores suelen denominarla "rancho".

En la segunda etapa, la vivienda se va transformando en una construcción con materiales más resistentes, mayor número de ambientes y dotada de servicios básicos, lo que redunda en una mejor calidad y condiciones mas favorables para su habitabilidad. Sus habitantes se refieren a ella como "casa" y representa un lugar seguro donde vivir, así como una inversión que conjuga una serie de esfuerzos y recursos.

Finalmente, en la tercera etapa, se produce en mayor o menor grado, la consolidación de la vivienda dentro de la comunidad. La estructura ha sido notablemente desarrollada, al tiempo que la vivienda ha sido equipada y dotada de determinadas comodidades. Adicionalmente a ello, ha sido el lugar donde se han criado los hijos y se ha formado la familia, y las personas se refieren a ella como "hogar".

2. Las diferentes fases del proceso constructivo del barrio. Dentro de este proceso, en forma similar al proceso de construcción de la vivienda, se derivan diferentes etapas:

a.La invasión del terreno, lo cual incluye el asentamiento inicial, caracterizado por los primeros trabajos de acondicionamiento del terreno; 
b. La instalación de los servicios básicos que favorezcan la habitabilidad y el funcionamiento de las viviendas y

c. La consolidación del barrio, integrando todos los servicios, o al menos parte de ellos. (Wiesenfeld, en prensa).

3. Los procesos grupales y psicosociales. Estos procesos se originan y desarrollan conjuntamente en las etapas de construcción mencionadas anteriormente. Los mismos obedecen a los aspectos vinculados a una construcción de una unidad social (el barrio), que resultan necesariamente inseparables de la construcción física del barrio y que se asocian con conceptos tales como la membrecía, la pertenencia, la identidad,y en general con todos los componentes del sentido de comunidad. (García, Giuliani \& Wiesenfeld, 1994).

Estos tres primeros temas comportan cada uno por su parte la actualización de tres etapas que sólo desde el punto de vista analítico pueden verse sustraídas del proceso general que desemboca en la consolidación del barrio. En este sentido, el paso del rancho al hogar, de la casa al barrio y el establecimiento de un sentido de comunidad fundado en las relaciones grupales, marchan conjuntamente en un proceso dinámico, transaccional e interdependiente que crea las condiciones de posibilidad para que tenga lugar la resistencia al desalojo o en todo caso posibilita la construcción de estrategias permiten a los habitantes lidiar con la amenaza de desalojo, cuestión esta que constituye el cuarto tema distinguido.

4. La amenaza del desalojo. Este tema tiene una presencia casi constante que surge a partir del momento de la invasión inicial y se mantiene a lo largo de las diferentes etapas de construcción de la comunidad . Así lo evidencian las permanentes referencias que las personas hacen respecto a las sucesivas amenazas de desalojo que enfrentó el barrio a través de su historia. Los testimonios aportados describen, por una parte, las respuestas organizativas que los pobladores generaron en cada situación de amenaza y, por la otra, se refieren a los aspectos emocionales que impregnaron estas situaciones. Es así, como pudimos detectar a través del análisis de la información suministrada, la forma en que la amenaza de desalojo, origina una serie de experiencias que repercutirán tanto sobre las etapas y procesos 
mencionados, como en el significado que le atribuyen los pobladores a sus viviendas, barrio y comunidad. (Wiesenfeld, 1995).

A continuación nos centraremos, en el análisis de aquellos aspectos que tienen que ver con las dificultades y contradicciones, así como con los beneficios que derivan de la interacción que se establece entre los vecinos y entre estos y las autoridades que intentan desalojarlos, en el proceso de construir un rancho y posteriormente consolidar un barrio y mejorar las viviendas. Con ello pretendemos ilustrar las transformaciones que a nivel personal, grupal y ambiental se van dando en los modos de hacer frente a la necesidad y a la ilegalidad para unos y otros. Para ello nos apoyaremos en citas textuales extraídas de 14 entrevistas en profundidad con pobladores del barrio.

4.1. La emoción como elemento movilizador. El temor es un sentimiento que está presente en los autoconstructores de ranchos y barrios. Previo a la invasión del terreno, donde intentarán erigir un techo, temen no tener un lugar en el que pernoctar, cuando invaden un terreno, temen la agresión de las autoridades, cuando construyen un rancho temen ser desalojados, cuando consiguen los servicios temen que se los quiten. Este miedo de ver diluida una esperanza por la intervención de las autoridades, induce a los autoconstructores a implementar estrategias tendientes a "ocultar" sus acciones:

Generalmente era de noche que se comenzaba,

y a realizarlas con un carácter de provisionalidad:

Los ranchos cuando se construyeron jamás se comenzó por hacer un rancho de bloques, por el mismo problema que había de la prohibición de hacer ranchos. Cada uno hacía los ranchos de cartón o de lata.

La dualidad entre la necesidad y el cumplimiento de la ley explica en parte este abordaje.

Ellos tenían razón pero nosotros también en cuanto a que no teníamos vivienda.

4.2. Cuando la emoción se transforma en acción, lo privado se hace público. Sin embargo, a medida que avanza el proceso de consolidar una vivienda este temor sufre transformaciones. En efecto, la concepción del problema de la vivienda de "mi familia" como un 
asunto que corresponde al ámbito de lo privado, se instala en el nivel de la conciencia de la necesidad individual, pero las prácticas sociales tendientes a satisfacerla suscitan encuentros, intercambios, negociaciones con otros que comparten la misma carencia y en el curso de dichas acciones e interacciones algo importante ocurre. Se dan cuenta que en la medida que el número de personas y de ranchos aumenta, se hace cada vez más difícil para las autoridades desplazarlos del lugar y que este incremento es posible gracias a las redes sociales que emergen ante la necesidad de unir esfuerzos para hacer frente a la "justicia":

Cuando hay un problema en el que todos sufrimos las mismas consecuencias entonces hay como mucha humanidad. Bueno, mientras construíamos había quienes cuidaban: No claveteen que viene la policía, perfecto, agáchense, apaguen las velas...en principio nos alumbrábamos con velas.

Esta complicidad espontánea, fomenta una suerte de cooperación basada en la competencia sana: ver quien construye más rápido como una manera de entorpecer las labores de desalojo,

Procurábamos batir récord. Nos hacíamos estas preguntas: 'Mira, cuantas horas duraste haciendo tu rancho inicial?' 'Yo lo hice en dos horas y media', 'Bueno yo voy a procurar hacerlo en dos horas.

Esta estrategia se complementa con el chantaje emocional a la autoridad. Ya no es posible ocultar lo evidente, de modo que implementan mecanismos que lo justifiquen, los cuales adoptan formas de manipulación y persuasión, en las que el manejo de la emocion es fundamental.

Cuando venía la autoridad: 'Mira que no se puede construir' 'Si, pero yo tengo ahí a mi mujer y mis muchachos'. Abrían y ahí estaba una mujer acostada en una cama con un muchachito. 'La verdad que es inhumano sacarlos' Y eso fue la forma de combatir las autoridades, combatirlos por el lado sentimental.

Los mecanismos empleados para convencer a la autoridad también se reflejan en la forma de construcción:

La mejor forma era adosando un rancho al lado del que ya estaba construido y cuando llegaba alguien: 'Mira que construyeron otro rancho' 'No, no, este no es un rancho más, esta es una habitación que yo le estoy haciendo a mi casa'. Este que ya estaba radicado aquí 
me defendía a mi que estaba haciendo el que estaba al lado. Luego se adosaba uno mas al otro lado. A la larga se formaba una tira de casas larguísima.

Interpretan que las autoridades son seres humanos sujetos a contradicciones y es la ambivalencia que detectan en ellos entre el deber y la solidaridad con "gente como ellos" en lo que se afianzan para proseguir. En efecto, se construye entre autoridades e invasores una suerte de intersubjetividad sustentada en factores que desbordan los límites de la relación impuesta por la situación que explícitamente deben resolver. Esto es, la complicidad que se establece entre los pobladores se traslada a su relación con las autoridades y es en el café, la cerveza, donde las tensiones, la manipulación y la coacción dan paso a la comprensión mutua.

La conformación de un grupo y de sus ranchos representa una construcción no sólo física sino también social y psicológica. El miedo se ha disipado y se ha transformado en fortaleza, en espíritu de lucha, en trazarse metas colectivas en las que los esfuerzos ya trascienden la esfera privada y son abiertos. Ya no es el ocultamiento ante el policía sino la demanda de los servicios a los organismos competentes.

Cuando éramos un grupo, un núcleo mas o menos, empezamos a luchar para que se le pusieran servicios al barrio, cloacas, agua, luz. Luego un organismo nos ayudó y metió las cloacas. Después se luchó para que nos metieran la luz que nosotros estábamos dispuestos a pagarla .. Fue una lucha inteligente, no como la de esos tiradores de piedras, no, no. Fue una lucha dirigiéndonos a quien nos podía oír. Dirigiendo cartas, dirigiéndonos personalmente a los organismos que nos podían solucionar el problema. En ningún momento hicimos como van muchos a tirarle piedras al policía que muchas veces es el que menos culpa tiene.

Las acciones abiertas hacia los organismos (cartas, reuniones) reflejan la apertura hacia otras formas de lucha en las que lo privado empieza a hacerse público y lo encubierto se hace manifiesto. Este cambio pareciera revelar la transición de una conciencia de la necesidad a una conciencia del derecho, de la concepción de la vivienda como refugio a la de vivienda como hogar, de la búsqueda de supervivencia a la lucha por mejorar la calidad de vida. 
En síntesis, la conjunción de los procesos que caracterizan los tres primeros temas de este análisis y la construcción de los procesos que conforman este último tema da con la siguiente paradoja: la amenaza de desalojo atenta insistentemente contra la consolidación del barrio, de manera frontal cuando se intenta construir el rancho y de manera diferida cuando se plantean reivindicaciones relacionadas con la obtención de servicios públicos, pero esta misma amenaza contribuye al mismo tiempo a fortalecer o afianzar el proceso de resistencia al desalojo al constituirse en núcleo de oposición que exige de los habitantes del barrio una cierta consecuencia, una cierta insistencia, (que adopta las formas de las respuestas emocionales y organizativas ya referidas) respecto de las metas dirigidas hacia la consolidación misma del barrio.

\section{Reflexiones finales}

El análisis da cuenta de la vinculación dinámica que existen entre todas las etapas y procesos señalados, al punto que podríamos decir que la construcción física y social de la vivienda y el barrio, pueden ser vistos como una construcción simultánea, formando parte de un mismo proceso. Ello resulta evidente, cuando se comprende que una vez invadido el terreno, la subsistencia y posterior consolidación de las viviendas, solo es posible en la medida que exista un entorno seguro y que al mismo tiempo cuente con los servicios básicos de funcionamiento. Ambos procesos se orientan a satisfacer necesidades conjuntas de los pobladores, lo cual genera una respuesta colectiva que abarca el quehacer en torno tanto a la vivienda como al barrio y la comunidad, por lo que no resulta extraño que el significado de ambos conceptos, aún teniendo su propia especificidad, mantengan una estrecha vinculación.

Todos estos procesos complejos, en lo que lo racional se complementa con lo afectivo, lo individual con lo colectivo, lo necesario con lo deseable, ocurren a espaldas de la ley pero en conjunción con ella, al margen de la planificación urbana pero paralela a ella. Lo descrito revela asimismo, que con las limitaciones del caso, los ciudadanos en los barrios construyen un orden social que no por diferente debe ignorarse. No se trata de idealizar los barrios y 
desconocer los problemas que allí ocurren. Al contrario, se trata de aceptarlos pero reconociendo que los problemas están igualmente presentes en la llamada ciudad formal y que así como los hay en ésta, en los barrios también existen valiosos recursos, importantes logros y numerosas potencialidades que merecen reivindicarse y sus habitantes disfrutar de los beneficios del espacio urbano de los que están excluidos. El reto que se impone entonces, es el desarrollo de un proyecto urbano que tomando en cuenta estos aspectos privilegie la inclusión a la segregación.

En la actualidad lo que ocurre es que desde el sector oficial, emanan programas sociales que decretan, incluso frecuentemente con las mejores intenciones hacia los beneficiarios, los cambios que afectarán su hábitat y modos de vida.

La dinámica de los barrios a su vez acarrea situaciones que ameritan profunda reflexión. Así, el asentamiento en terrenos geológicamente inestables en los que los servicios se congestionan y afectan aún más su vulnerabilidad, conduce a vivir en situaciones de riesgo de derrumbe que no pocas veces acarrean pérdidas humanas, sin escatimar las materiales.

Ambas situaciones revelan estrategias de acción en las que los dos tipos de agentes involucrados, actúan uno al margen del otro. Así, en el caso de las políticas estatales, los gestores de las mismas las elaboran suponiendo un conocimiento acerca de las necesidades, deseos, hábitos de los sujetos a quienes las mismas van dirigidas, sin considerar las opiniones de estos, quienes son en última instancia los que justificarán o no dichas políticas, con las fuertes inversiones que ellas acarrean. Ello sin desconocer que el mero acto de formular estas políticas constituye en sí una contradicción, ya que implícitamente se avala lo que en principio se niega, a saber: la consolidación de barrios de ranchos. Por su parte, el habitante de barrio, ante el dramatismo de sus condiciones de supervivencia, improvisa soluciones que pueden revertir en su propia desgracia.

Ambas posturas reflejan profundas contradicciones. Del lado de los organismos oficiales, ésta radica en generar soluciones para un protagonista que desconocen desde su perspectiva de no-habitante de 
barrio, y al que inducen a vivir de cierta manera que le es ajena, en virtud de su contexto e idiosincrasia. La contradicción radica en indicar un que y como para un personaje cuya voz es la que debe orientar dicho que y como. Una cita que ilustra este procedimiento, es la expresada por Sennett (1975), quien afirmó: “Una y otra vez puede escucharse en las esferas de planificación un temor explícito cuando los seres humanos afectados por los cambios planificadores se interesan, siquiera levemente en los remedios propuestos para sus modos de existencia" (p. 30).

Desde el lado del barrio, la contradicción emana del hecho que en el deseo de sus habitantes por incorporarse dentro de la vida urbana, se automarginan de ella, al fomentar acciones que van en detrimento de los servicios en la "ciudad formal" y de la seguridad de los habitantes en los propios barrios.

Con base en las consideraciones expuestas, sustentamos que se requiere un cambio de enfoque hacia el desarrollo de la "ciudad informal". En este cambio las necesidades que se identifiquen como prioritarias y los procedimientos que se establezcan para su satisfacción deben ser el resultado de la acción conjunta de los gerentes sociales y de los usuarios, de modo tal que mediante la comunicación continua ambas partes reconozcan sus limitaciones, sus recursos y los requerimientos culturales que deben respetar las decisiones que se adopten. Un programa de este tipo requiere de una metodología de trabajo que tomando las posibilidades de inversión de la gerencia pública, potencie desarrollos adicionales de la población como lo es su organización y su reconocimiento como ciudadanos que forman parte activa de la trama social urbana, y sobre todo reivindique el componente psicosocial que proyectos como estos entrañan. Tal como se evidenció en páginas anteriores, la emocion representa una dimensión que por su protagonismo en este tipo de procesos no puede ser escatimada. Es una característica cultural y como tal debe ocupar un lugar importante en el proceso de incorporación urbana de los sectores tradicionalmente desincorporados de este contexto. En otras palabras, esta propuesta exige que los agentes externos conciban este tipo de trabajo como uno "en" y "con" las comunidades, en vez de 
enfocarlo como una gestión "para" las mismas. Esta distinción aparentemente inocente reorienta una práctica ejercida desde las esferas del poder que implícitamente descalifica al otro, al impedir incorporar su voz a lo largo del proceso de toma de decisiones orientadas a generar cambios supuestamente deseables para él, como si tuviese la potestad de ponerse en el lugar del otro sin dejar de lado su rol de experto. Las comunidades a su vez, requieren problematizar situaciones que forman parte de su cotidianidad y que por tanto han llegado a considerar naturales, a fin de generar una reflexión acerca de su posición dentro de la sociedad y de las razones de la misma, que promueva la realización de acciones tendientes a superar la desesperanza ante una mejor calidad de vida. En síntesis, de superar la polaridad yo vs. el otro y propiciar el encuentro entre (Fine, 1994) o intersubjetividades (Fernández-Christlieb, 1994). En términos de Sennett (1975): "Esta es la lección que el sector planificador necesita ahora aprender: debe asumir la responsabilidad de sus actos en una sociedad histórica e imprevisible, más bien que en un mundo soñado de armonía y orden predeterminado.” (p. 118).

\section{Referencias}

Blumer, H. (1969). Symbolic interactionism: perspective and method. Englewood Cliffs: Prentice Hall.

Bolivar, T., Guerrero, M., Rosas, I.-., Ontiveros, T., De Freitas, J., Arnal, H., \& Sancio.R. (1994). Densificación y vivienda en los barrios caraqueños. Caracas: Consejo Nacional de la Vivienda.

Bruner, J. (1991). Actos de significado: mas allá de la revolución cognitiva. Madrid: Alianza.

Duhau, E. (1993). Urbanización popular y orden urbano. En G. Fadda Cori (Comp.), La urbe latinoamericana: Balance y perspectivas a las puertas del tercer milenio (pp.237-252). Caracas: Universidad Central de Venezuela, Consejo de Desarrollo Científico y Humanístico.

Fernández-Christlieb, P. (1994). Psicología social, intersubjetividad y psicología colectiva. En M. Montero (Coord.), Construcción y crítica de la psicología social (pp.49-107). Barcelona: Anthropos.

Fine, M. (1994). Working the hyphens. Reinventing self and other in qualitative research. En N. Denzin \& Y. Lincoln (Comps.), Handbook of qualitative research (pp.70-82 ). Los Angeles: Sage.

García, I., \& Giuliani, F. (1992). La noción de comunidad y el sentido de comunidad en un barrio caraqueño. Tesis de Grado, Escuela de Psicología, Universidad Central de Venezuela, Caracas. 
García, I., Giuliani, F., \& Wiesenfeld, E (1994). El lugar de la teoria en psicología social comunitária: comunidad y sentido de comunidad. En M. Montero (Comp.), Psicología social comunitária (pp. 75-102). Guadalajara, México: Universidad de Guadalajara.

Gergen, K. (1985). Social constructionist inquiry: Context and implications. En K. Gergen \& K. Davis (Comps.), The social construction of the person (pp.3-18). Nueva York, Springer Verlag.

Giuliani, F. (1996). La reconstrucción critica de la historia de un barrio caraqueño. Universidad Central de Venezuela, Caracas, manuscripto no publicado.

Guitián, D. (1993). Espacio habitable popular de la Caracas contemporánea. En G. Fadda Cori (Comp.) La urbe latinoamericana: Balance y perspectivas a las puertas del tercer milenio (pp.89-118). Caracas: Universidad Central de Venezuela, Consejo de Desarrollo Científico y Humanístico.

Guba, E. (1990). The alternative paradigm dialog. En E. Guba (Comp.) The paradigm dialog. London: Sage.

Harré, R. (1989). La construcción social de la mente: la relación íntima entre el lenguaje y la interacción social. En T. Ibañez (Coord.) El conocimiento de la realidad social (pp.39-52). Barcelona: Sendai.

Ibañez, T. (1989). La psicología social como dispositivo desconstruccionista. En T. Ibañez (Coord.) El conocimiento de la realidad social (pp.109-133). Barcelona: Sendai.

Ibañez, T. (1994). Construccionismo y psicología. Revista Interamericana de Psicología, 28,(1), 105-123.

Jimenez, B. (1993). Villas de San Juan: satisfacción residencial y calidad de vida en Guadalajara. Ponencia presentada en el XXIV Congreso Interamericano de Psicología, Santiago de Chile, 4-9 de Julio, 1993.

Lincoln, Y. (1994). Emergent paradigms and the crisis in psychology. Revista Interamericana de Psicología, 28,(2), 139-154.

Lovera, A. (1989). El deterioro de las condiciones urbanas en Venezuela. En M. Schteingart (Comp.). Las ciudades latinoamericanas en la crisis. Problemas y desafios. México: Trillas.

Negrón, M. (1993). Ciudad "formal” y ciudad “informal”. Una sola ciudad. En G. Fadda Cori (Comp.) La urbe latinoamericana: Balance y perspectivas a las puertas del tercer milenio (pp.255-266). Caracas: Universidad Central de Venezuela, Consejo de Desarrollo Científico y Humanístico.

Ruibal, H. (1993). La comunidad urbana y la vivienda productiva. Sugerencias para el desarrollo de actividades productivas como eje del mejoramiento de los asentamientos urbanos pobres. En B. Kliksberg (Comp.) ¿Cómo enfrentar la pobreza? México: Grupo Editor Latinoamericano.

Schteingart, M. (1993). Informalidad, empleo y vivienda en América Latina (con especial referencia al caso de México). En G. Fadda Cori (Comp.) La urbe latinoamericana: balance y perspectivas a las puertas del tercer milenio (pp.157176). Caracas: Universidad Central de Venezuela, Consejo de Desarrollo Científico y Humanístico.

Sennett, R. (1975). Vida urbana e identidad personal. Barcelona: Edicions 62.

Taylor, S., \& Bogdan, R. (1986). Introducción a los métodos cualitativos de investigación: la búsqueda de significados. Buenos Aires: Paidós. 
Wiesenfeld, E. (1995). La vivienda: su evaluación desde la psicología ambiental. Caracas: Universidad Central de Venezuela, Consejo de Desarrollo Científico y Humanístico.

Wiesenfeld, E. (En prensa). The construction of the meaning of a barrio house. Environment and Behavior.

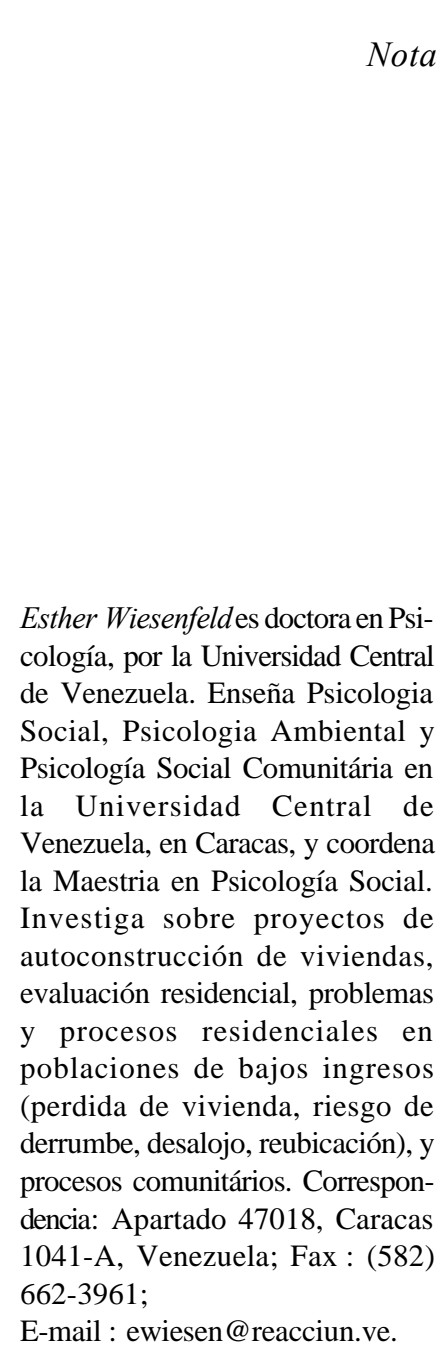

${ }^{1}$ Ponencia presentada en el XXV Congreso Interamericano de Psicología, San Juan de Puerto Rico, del 9 al 14 de Julio de 1995.

Sobre a autora 